\section{Identification of biallelic germline variants of SRP68 in a sporadic case with severe congenital neutropenia}

Congenital neutropenia $(\mathrm{CN})$ are a heterogeneous group of very rare diseases characterized by profound and chronic neutropenia possibly associated with comorbidities. ${ }^{1}$ Variants in about 25 genes implicating distinct pathophysiological pathways have been identified in CN. ${ }^{1}$ Recently, co-translational targeting of nascent secretory and membrane proteins to the endoplasmic reticulum (ER) mediated by the signal recognition particle (SRP) complex has been implicated in the pathogenesis of both $\mathrm{CN}$ and Shwachman-Diamond-like syndrome. ${ }^{2,3}$ In humans, the SRP complex is composed of six proteins (SRP9, SRP14, SRP19, SRP54, SRP68, and SRP72) assembled on a 7S RNA molecule. ${ }^{4}$ The SRP54 protein with its GTPase activity constitutes the key element of this complex and regulates the entire physiological process. ${ }^{5} \mathrm{We}$ recently reported germline SRP54 variants in a large number of sporadic and familial cases with autosomal dominant $\mathrm{CN}^{3}{ }^{3}$ Apart from SRP54, germline heterozygous variants of $S R P 72$ have been described in a few cases with aplastic anemia and myelodysplasia. ${ }^{6,7}$

Here, we report a sporadic case of severe $\mathrm{CN}$ associat- ed with biallelic pathogenic variants of SRP68, implicating for the first time the SRP68 protein in the pathogenesis of CN. Specifically, we investigated the functional consequences of SRP68 defect on granulopoiesis and on ER homeostasis (see the Online Supplementary Appendix). The patient is a Caucasian boy referred for an anal abscess at 6 weeks of age. Blood counts showed severe neutropenia (white blood cells $6.1 \times 10^{9} / \mathrm{L}$, neutrophils $\left.0.2 \times 10^{9} / \mathrm{L}\right)$, elevated monocyte count $\left(1.7 \times 10^{9} / \mathrm{L}\right)$, anemia (hemoglobin $7.5 \mathrm{~g} / \mathrm{dL}$ ) associated with iron deficiency and moderate thrombocytopenia (platelets $149 \times 10^{9} / \mathrm{L}$ ). Serial blood counts $(n=41)$ confirmed the persistent and profound neutropenia $\left(0.200 \times 10^{9} / \mathrm{L}\right.$ [range: $\left.\left.0-1.800\right]\right)$. The bone marrow examination showed a maturation arrest at the promyelocytic stage and major features of dysgranulopoiesis. Promyelocytes displayed numerous condensed granulations, abnormal nuclei, clumped chromatin and the absence of cytoplasmic vacuoles; the remaining neutrophils were highly dystrophic (Figure 1A). In the first year of life, neutropenia was associated with profound anemia and thrombocytopenia which recovered later. The level of hemoglobin and the platelet count were respectively $8.7 \mathrm{~g} / \mathrm{dL}$ [range, 5.5-11.3] and $132 \times 10^{9} / \mathrm{L}$ [range, 43-318]). From diagnosis to the last follow-up at the age of 5 years, he had a prophylactic antibiotherapy and was treated with granulocyte colony-stimulating fac-

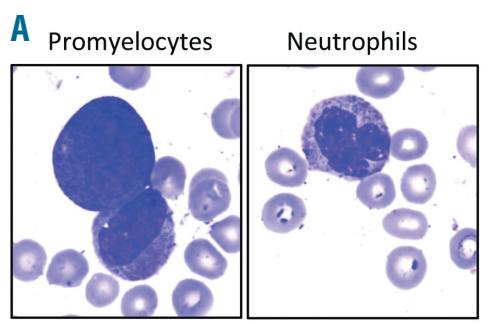

Patient's bone marrow

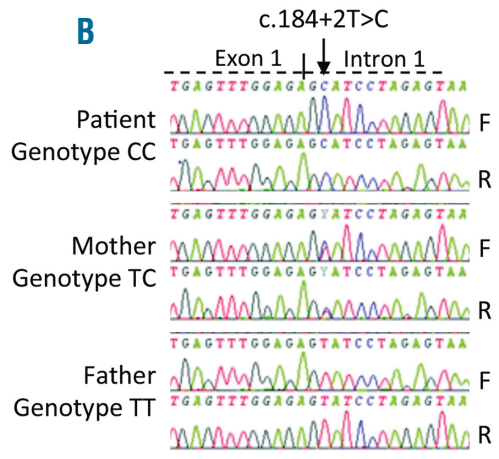

C

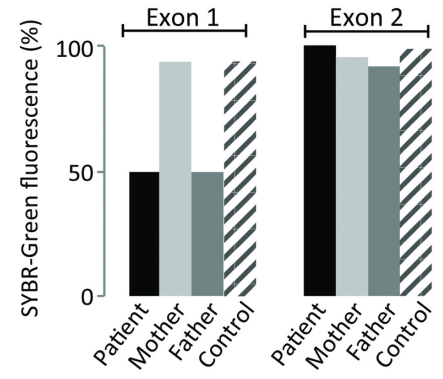

D

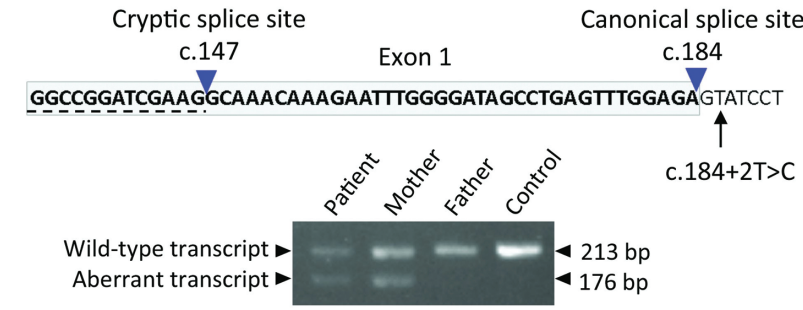

Cryptic splice site _. Exon 1. . . . . . . Exon 2 . . . . . .

Aberrant transcript GGCCGGATCGAAGTTCTTCA GATTATTA sequence

Figure 1. A sporadic case with severe congenital neutropenia and loss-of-function variants of SRP68. (A) Cytology analysis after May Grunwald-Giemsa staining of patient's bone marrow. Pictures represent granulocytic precursors (left) and neutrophils (right) (original magnification $\times 100)$. (B) Sanger sequencing confirmation of the splice site variant c.184+2T>C located in intron 1 of SRP68. F: forward strand; R: reverse strand. (C) Confirmation of the deletion of exon 1 of SRP68 by quantitative polymerase chain reaction (PCR) using SYBR-Green. (D) Predicted alternate cryptic splice site located 37 bp upstream of the splice donor site shown on SRP68 sequence and confirmed by RT-PCR using RNA extracted from fibroblasts and Sanger sequencing of the shorter product (176 bp). (E) Expression level of SRP68 protein in fibroblasts evaluated by western blot analysis 
A

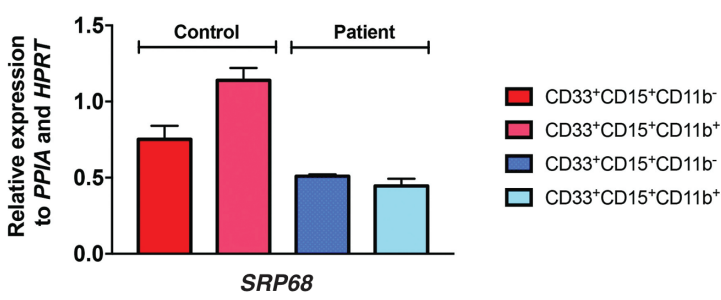

B

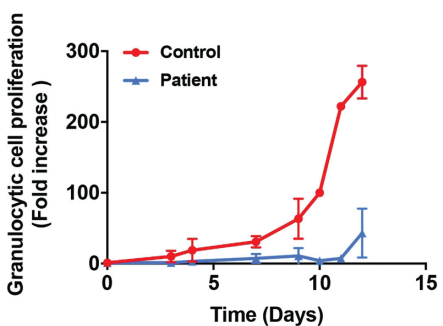

C

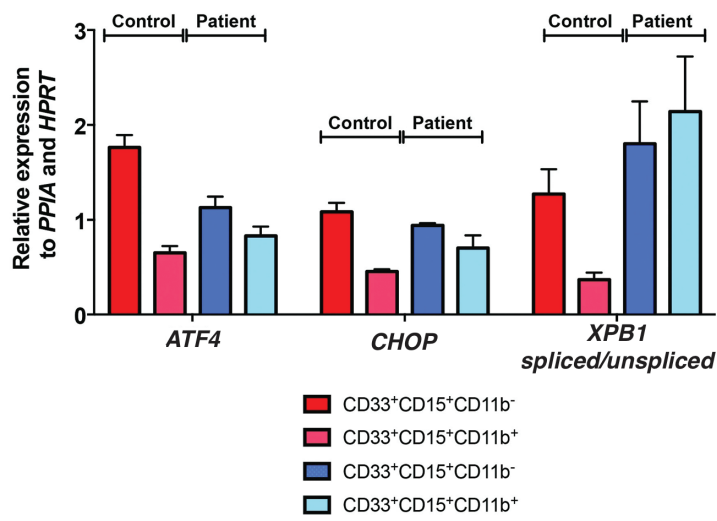

D

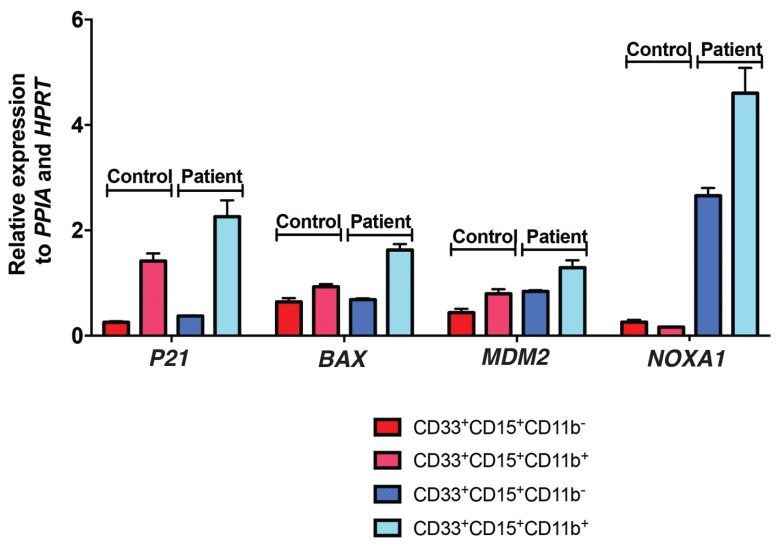

Figure 2. SRP68 biallelic variants induce a defect in granulocytic differentiation and endoplasmic reticulum stress and increase P53-dependent apoptosis of granulocytic precursors and neutrophils. CD34 ${ }^{+}$cells from the patient and the control donor were purified from blood and cultured for 12 days in serum freemedium with stem cell factor, interleukin 3 and granulocyte colony-stimulating factor. Sorted granulocytic cells $\mathrm{CD}^{2} 3^{+} 15^{+} 11 \mathrm{~b}^{-}$and $\mathrm{CD} 33^{+} 15^{+} 11 \mathrm{~b}^{+}$were sorted on day 12. Expression levels were determined by quantitative everse transcription polymerase chain reaction related to PPIA and HPRT on day 12 . (A) Expression level of SRP68 ( $n=1$ in triplicate; mean \pm standard deviation [SD]). (B) Fold increase in cell proliferation during granulocytic differentiation ( $n=2 ;$ mean $\pm S D)$. (C) Expression level of ATF4, CHOP and ratio spliced/unspliced XBP1 ( $\mathrm{n}=1$ in triplicate; mean $\pm \mathrm{SD}$ ). (D) Expression level of $P 21, B A X, M D M 2$ and NOXA1 ( $=1$ in triplicate; mean $\pm S D$ )

tor (5 to $10 \mu \mathrm{g} / \mathrm{kg} / \mathrm{day})$. With this treatment and despite the persistent severe neutropenia, the patient has not presented severe bacterial infections. There was no familial medical history. After excluding the genes classically involved in $\mathrm{CN}$ by targeted high throughput sequencing, we performed whole exome sequencing (WES) on a triobased approach (Online Supplementary Appendix). WES diagnosed an intronic homozygous point substitution affecting the splice donor site of exon 1 (c.184+2T>C) of the SRP68 gene. This variant was detected at a heterozygous state in his mother and was absent in his father suggesting the presence in trans of a large deletion (Online Supplementary Table S1). Sanger sequencing and quantitative polymerase chain reaction (PCR) confirmed the splice site variant and the deletion of exon 1 of SRP68 (Figure 1B-C; Online Supplementary Table S2). This latter was inherited from his father confirming the compound heterozygous SRP68 genotype (c.184+2T>C) (exon 1 deletion) of the patient.

In order to determine the consequences of the intronic SRP68 variant on splicing, we performed reverse transcription PCR (RT-PCR) using primary fibroblasts from the patient, his parents, and a control donor. Agarose gel electrophoresis indicated that the patient and his mother harbor both the expected $213 \mathrm{bp}$ fragment and a shorter product (176 bp). Sequencing of this fragment revealed the use of a predicted cryptic splice donor site located 37 bases upstream (c.147) and resulting in the premature truncation of the mutated protein (Ala50Phefs*52) (Figure 1D). By western blot analysis, we found a drastic decrease of SRP68 protein expression in fibroblasts of the patient compared to his parents (Figure 1E) demonstrating the loss-of-function effect of the SRP68 defects. Nevertheless, we observed a residual protein expression in the patient that may be due to a partial splicing defect as suggested by the semi-quantitative analysis of SRP 68 transcripts in the mother (ratio $65 \% / 35 \%$ for both 213 $\mathrm{bp} / 176 \mathrm{bp}$ fragments, Figure 1D). This finding was also consistent with the SRP68 transcript expression level determined from patient's granulocytic cells. The residual expression of SRP68 could be explained by the specific post-transcriptional splicing mechanism related to GT>GC variants affecting the canonical 5' donor splice site as identified in our patient (c.184+2T>C). In fact, $5^{\prime}$ splice site GT>GC variants may retain their ability to generate normal transcripts and be associated with a milder than expected clinical phenotype. SRP68 functions only as a heterodimeric structure with the SRP72 protein..$^{9}$ We could speculate that null SRP68 variants would be lethal on the basis of what was recently shown in $\operatorname{Srp} 72^{-/-}$mice. ${ }^{10}$

We purified $\mathrm{CD}_{3} 4^{+}$progenitor cells from peripheral blood and cultured them in serum-free medium supplemented with stem cell factor $(25 \mathrm{ng} / \mathrm{mL})$, interleukin 3 $(10 \mathrm{ng} / \mathrm{mL})$ and granulocyte colony-stimulating factor (20 $\mathrm{ng} / \mathrm{mL}$ ) for 12 days. The level of SRP68 expression on day 
12 was reduced to $68 \%$ in immature $\mathrm{CD}_{3}{ }^{+} \mathrm{CD} 15^{+} \mathrm{CD} 11 \mathrm{~b}^{-}$cells and to $39 \%$ in the more mature $\mathrm{CD} 33^{+} \mathrm{CD} 15^{+} \mathrm{CD} 11 \mathrm{~b}^{+}$cells of the patient compared with control cells (Figure 2A). We also analyzed the consequences of SRP68 defect on granulocyte proliferation. In the same experimental conditions, we cultured CD34 ${ }^{+}$cells purified from blood samples and observed nearly six-times less granulocytic cell proliferation in the patient compared to $\mathrm{CD} 34^{+}$cells from healthy control (Figure 2B).

These results highlight the major role of the SRP complex during granulocytic differentiation. This physiological process requires the production and maturation of a huge number of granule proteins. ${ }^{11}$ This context explains why granulocytic precursors and neutrophils are highly sensitive to alterations of protein synthesis, protein transport into ER/Golgi and ER protein misfolding. A defective co-translational targeting of nascent proteins may either affect the level of ER resident proteins as chaperones and glycosylases or lead to incorrectly translocated proteins essential for granulocytic precursor maturation as the neutrophil elastase ELANE. ${ }^{12}$ Both hypotheses will result in ER stress, activation of the unfolded protein response (UPR) and finally, to cycle cell arrest, senescence and/or death by apoptosis or necrosis of granulocytic cells. In mammals, the SRP68/SRP72 heterodimer plays an essential role in the recognition of the signal peptide of nascent proteins and in their translocation through the SRP receptor located at the ER membrane. ${ }^{13}$ As we previously showed that SRP54 variants induce an ER stress and activate the UPR pathway, we investigated several markers of UPR activation (ATF4, CHOP and spliced XBP1). ${ }^{3}$ Using in vitro sorted granulocytic cells, we found a significant increase in spliced XBP1 expression level in both immature and mature granulocytic cells of the patient in comparison with control cells pointing out the specific activation of the IRE1 ER stress sensor of the UPR signaling (Figure 2C) ${ }^{14}$ In contrast to SRP54, no activation of the PERK pathway, another distinct ER sensor, was observed by analyzing ATF4 and CHOP UPR-target genes. ${ }^{14}$ Under ER stress conditions, the induction of UPR leads to enhanced apoptosis reducing the number of granulocytic precursors and neutrophils. In most $\mathrm{CN}$, including SRP54-related CN, apoptosis seems to be dependent on $\mathrm{p} 53$ pathway activation. ${ }^{3}$ We analyzed, by quantitative RT-PCR, the expression level of several P53 target genes (BAX,NOXA1, P21 and MDM2) in sorted granulocytic cells (Figure $2 \mathrm{D}$ ). The patient displayed a higher expression level of P53 target genes than in control and the activation was more pronounced in the more mature granulocytic $\mathrm{CD}_{3} 3^{+} \mathrm{CD} 15^{+} \mathrm{CD} 11 \mathrm{~b}^{+}$cells. However, we could not definitively exclude P53-independent pathways that also lead to cell cycle arrest and apoptosis. ${ }^{15}$ Of note, P53-independent nucleolar stress has been reported in animal models of bone marrow failure syndromes caused by impaired ribosomal biogenesis and function. ${ }^{15}$

Besides the profound neutropenia, this patient presented transient severe anemia and thrombocytopenia as also reported in patients harboring SRP54 and SRP72 defects. ${ }^{3,6}$ We could hypothesize that an aberrant SRP complex, regardless of the implicated defective SRP protein, may have consequences on the targeting of proteins essential for hematopoietic stem cells or erythroid, megakaryocytic and granuloytic progenitor/precursors. Further studies are needed to characterize the mechanisms leading to impaired differentiation of hematopoietic lineages and to identify the protein partners of the SRP complex.
In conclusion, we have identified a novel genetic entity of CN affecting another protein of the SRP complex underlying the major implication of the universally conserved co-translational targeting machinery of proteins in the pathogenesis of $\mathrm{CN}$. These first observations show that loss-of-function SRP58 variants trigger an ER stress resulting in an increased $\mathrm{P53}$-dependent apoptosis of granulocytic precursors and neutrophils.

Barbara Schmaltz-Panneau, ${ }^{1,2}$ Anne Pagnier, ${ }^{3}$

Séverine Clauin, ${ }^{4}$ Julien Buratti, ${ }^{3}$ Caroline Marty, ${ }^{1,2}$

Odile Fenneteau, ${ }^{5}$ Klaus Dieterich, ${ }^{6}$ Blandine Beaupain, ${ }^{7,8}$

Jean Donadieu, ${ }^{7,8,9}$ Isabelle Plo ${ }^{1,2}$

and Christine Bellanné-Chantelot ${ }^{1,4,8}$

${ }^{1}$ Gustave Roussy Cancer Center, INSERM U1287, Villejuif; ${ }^{2}$ Paris Saclay University, U1287, Villejuif; ${ }^{3}$ Department of Pediatric Hematology and Oncology, CHU Grenoble Alpes, Grenoble, GIN, Grenoble; ${ }^{4} A P-H P$, Pitié-Salpêtrière Hospital, DMU BioGeM, Department of Genetics, Sorbonne University, Paris; ${ }^{5}$ AP-HP, Robert Debré Hospital, Laboratory of Hematology, University of Paris, Paris; ${ }^{6}$ Department of Medical Genetics, Univsersity Grenoble Alpes, INSERM U1216, CHU Grenoble Alpes, GIN, Grenoble; ${ }^{7}$ French Registry of Chronic Neutropenia, Trousseau Hospital, Paris; ${ }^{8}$ Reference Center for Chronic Neutropenia, Paris and ${ }^{9}$ AP-HP, Trousseau Hospital, Department of Pediatric Hematology and Oncology, Paris, France

Correspondence: CHRISTINE BELLANNE'-CHANTELOT christine.bellanne-chantelot@aphp.fr

doi:10.3324/haematol.2020.247825

Disclosures: no conflicts of interest to disclose

Contributions: BSP performed most of the research and analyzed functional data; AP provided samples and clinical data; SC performed and analyzed molecular experiments; JB performed bioinformatics analysis; CM performed fibroblast culture and western blot; OF performed cytological analysis; KD provided samples and clinical data; $B B$ collected clinical data; JD involved in the clinical part and contributed to intellectual input; IP designed the study, analyzed the data and critically reviewed the paper; $C B-C$ designed the study, analyzed the data and wrote the paper.

Acknowledgments: the authors would like to thank the family involved in the study. The authors also thank the Cytometry Platform (PFIC) of Gustave Roussy, especially Philippe Rameau and Yann Lecluse.

Funding: the whole-exome sequencing was funded by the Foundation for rare diseases (AO9102LS) and the research was supported by grants from INCA-PLBIO 2017 (I Plo). The French Registry is supported by grants from X4 pharma, Prolong Pharma and Chugai $S A$ to $B B$ and JD.

\section{References}

1. Donadieu J, Beaupain B, Fenneteau O, Bellanne-Chantelot C. Congenital neutropenia in the era of genomics: classification, diagnosis, and natural history. Br J Haematol. 2017;179 (4):557-574.

2. Carapito R, Konantz M, Paillard C, et al. Mutations in signal recognition particle SRP54 cause syndromic neutropenia with Shwachman-Diamond-like features. J Clin Invest. 2017;127 11):4090-4103.

3. Bellanne-Chantelot C, Schmaltz-Panneau B, Marty C, et al. Mutations in the SRP54 gene cause severe congenital neutropenia as well as Shwachman-Diamond-like syndrome. Blood. 2018; 132(12):1318-1331.

4. Wild K, Juaire KD, Soni K, et al. Reconstitution of the human SRP system and quantitative and systematic analysis of its ribosome interactions. Nucleic Acids Res. 2019;47 (6):3184-3196.

5. Focia PJ, Shepotinovskaya IV, Seidler JA, Freymann DM. Heterodimeric GTPase core of the SRP targeting complex. Science. 2004;303(5656):373-377

6. Kirwan M, Walne AJ, Plagnol V, et al. Exome sequencing identifies autosomal-dominant SRP72 mutations associated with familial aplasia and myelodysplasia. Am J Hum Genet. 2012;90(5):888-892. 
7. Bluteau O, Sebert M, Leblanc T, et al. A landscape of germ line mutations in a cohort of inherited bone marrow failure patients. Blood. 2018;131(7):717-732.

8. Lin JH, Tang XY, Boulling A, et al. First estimate of the scale of canonical 5' splice site GT>GC variants capable of generating wild-type transcripts. Hum Mutat. 2019;40(10):1856-1873.

9. Gao Y, Zhang Q, Lang Y, et al. Human apo-SRP72 and SRP68/72 complex structures reveal the molecular basis of protein translocation. J Mol Cell Biol. 2017;9(3):220-230.

10. D'Altri T, Schuster MB, Wenzel A, Porse BT. Heterozygous loss of Srp72 in mice is not associated with major hematological phenotypes. Eur J Haematol. 2019;103(4):319-328.
11. Cowland JB, Borregaard N. Granulopoiesis and granules of human neutrophils. Immunol Rev. 2016;273(1):11-28.

12. Grenda DS, Murakami M, Ghatak J, et al. Mutations of the ELA2 gene found in patients with severe congenital neutropenia induce the unfolded protein response and cellular apoptosis. Blood. 2007; 110(13):4179-4187.

13. Elvekrog MM, Walter P. Dynamics of co-translational protein targeting. Curr Opin Chem Biol. 2015;29:79-86.

14. Walter $P$, Ron $D$. The unfolded protein response: from stress pathway to homeostatic regulation. Science. 2011;334(6059):1081-1086.

15. James A, Wang Y, Raje H, Rosby R, DiMario P. Nucleolar stress with and without p53. Nucleus. 2014;5(5):402-426. 